\title{
Images of the month 2: Pulmonary artery pseudoaneurysm formation within uterine leiomyosarcoma metastases
}

\author{
Authors: Divya Vaid, ${ }^{\mathrm{A}}$ Veronika Majcher, ${ }^{\mathrm{B}}$ Nicholas Heptonstall, ${ }^{\mathrm{C}}$ Timothy $]$ Sadler ${ }^{\mathrm{D}}$ and Andrew P Winterbottom ${ }^{\mathrm{E}}$
}
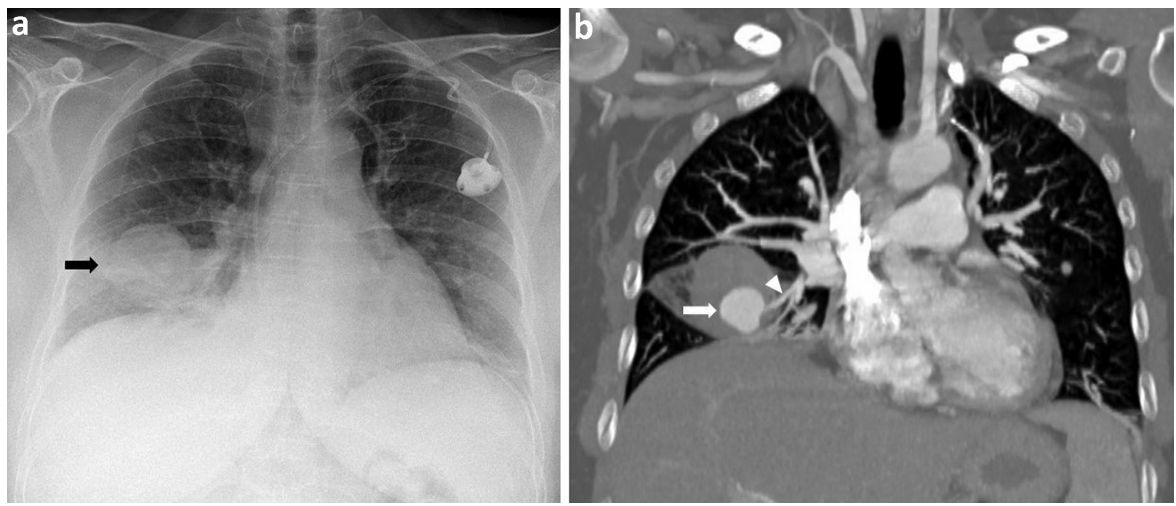

Fig 1. Chest imaging on admission. a) Chest $\mathrm{X}$-ray demonstrating a significant increase in size of the known right lower zone pulmonary metastatic lesion with surrounding hazy opacification (black arrow). b) Computed tomography pulmonary angiography showing a new $2.5 \mathrm{~cm}$ pulmonary artery pseudoaneurysm (white arrow) within the pulmonary metastasis in the right middle lobe arising from the lateral segmental branch of right middle lobe pulmonary artery (white arrowhead).

We present a rare case of pulmonary artery pseudoaneurysm formation in leiomyosarcoma metastases with evidence of acute bleeding and subsequent interventional radiological management.

KEYWORDS: pulmonary artery pseudoaneurysm, chest imaging, pulmonary metastases, interventional radiology, uterine leiomyosarcoma

DOI: $10.7861 /$ clinmed.2021-0494

\section{Case presentation}

A 46-year-old woman presented to the emergency department with a 2-day history of progressively worsening chest pain and shortness of breath. There was no haemoptysis. Background history was notable for high-grade uterine leiomyosarcoma diagnosed 10 years previously, for which she underwent total

Authors: A senior clinical fellow in radiology, Addenbrooke's Hospital, Cambridge, UK; ${ }^{\text {B }}$ specialty registrar in radiology, Addenbrooke's Hospital, Cambridge, UK; ' Cellow in interventional radiology, Addenbrooke's Hospital, Cambridge, UK; ${ }^{D}$ consultant radiologist, Addenbrooke's Hospital, Cambridge, UK; ${ }^{\text {E }}$ consultant interventional radiologist, Addenbrooke's Hospital, Cambridge, UK abdominal hysterectomy followed by local radiotherapy and adjuvant chemotherapy. She had been receiving hormone therapy for the last 3 years for disease progression. A computed tomography (CT) 2 months prior showed multiple lung metastases, which had increased in size along with a stable small right lower lobe pulmonary artery pseudoaneurysm (PAP). Relevant past medical history also included previous pulmonary embolism, for which she had completed a course of therapeutic anticoagulation 1 year previously. Following an initial chest X-ray, a contrast-enhanced CT pulmonary angiography (CTPA) was performed to rule out recurrent pulmonary embolism.

The chest $X$-ray demonstrated a significant increase in size of the known right lower zone pulmonary metastatic lesion with surrounding hazy opacification consistent with right middle lobe consolidation (Fig 1a). CTPA showed a new $2.5 \mathrm{~cm}$ pulmonary artery pseudoaneurysm within the pulmonary metastasis in the right middle lobe arising from the lateral segmental branch of right middle lobe pulmonary artery. There was surrounding haematoma and ground glass opacity in the right middle lobe in keeping with pulmonary haemorrhage (Fig 1b). These findings, along with a moderate right haemothorax, were consistent with a recent bleed. The right lower lobe pseudoaneurysm was stable.

She underwent successful embolisation of both the ruptured right middle lobe pseudoaneurysm and the stable right lower lobe pseudoaneurysm with insertion of a single vascular plug into each of the respective feeding segmental pulmonary arteries with no post-procedural complications (Fig 2a). A post-procedural CTPA performed prior to her discharge from the hospital demonstrated 
Fig 2. a) Digital subtraction right lung pulmonary angiography showing insertion of a single vascular plug into each of the respective feeding segmental pulmonary arteries leading to embolisation of both the ruptured right middle lobe pseudoaneurysm (black arrow) and the stable non-ruptured right lower lobe pseudoaneurysm (black arrowhead). b) Post-procedural computed tomography pulmonary angiography performed prior to discharge showing the vascular plug (white arrowhead) in the feeding segmental right middle lobe pulmonary artery with complete embolisation of the pseudoaneurysm.
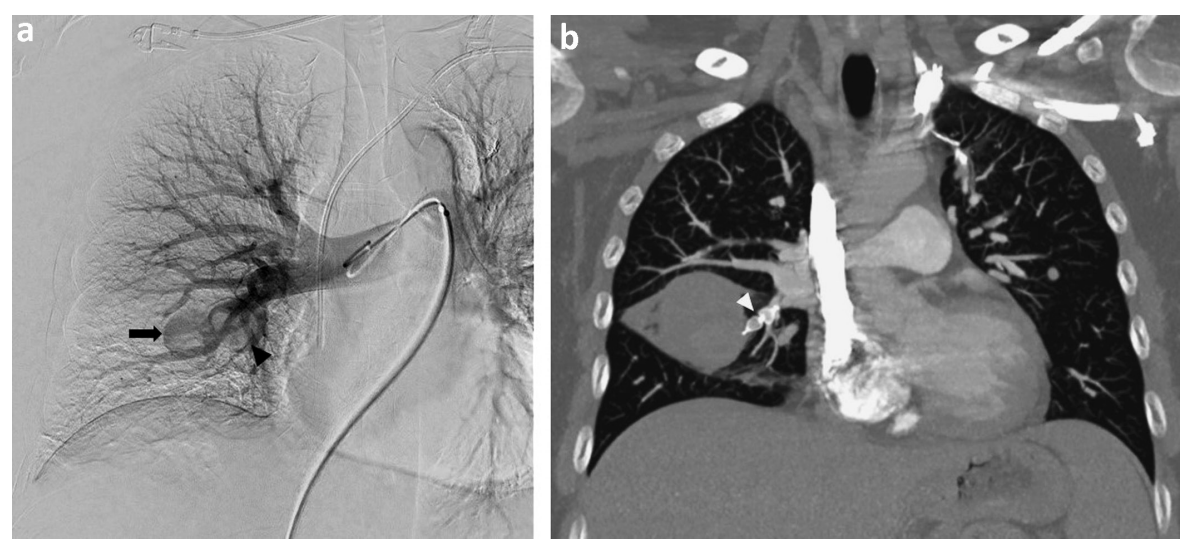

no further filling of the pulmonary arterial pseudoaneurysms (Fig 2b). On her most recent clinical follow-up, she remained asymptomatic from her disease with a normal performance status.

\section{Discussion}

Pulmonary artery pseudoaneurysm is a rare life-threatening entity that arises due to disruption of the pulmonary artery wall leading to contained rupture. ${ }^{1,2}$ The most common cause of PAP is infection, but cases of PAP secondary to neoplastic aetiology where the tumour is thought to directly invade the vessel wall have also been rarely reported. These include PAP within primary squamous cell carcinoma and adenocarcinoma of lung and metastases from angiosarcoma, renal cell carcinoma and cardiac myxofibrosarcoma among others. ${ }^{1-3}$ Due to its non-specific clinical presentation and low prevalence, PAP remains a diagnostic challenge for clinicians. Imaging has an important role in prompt recognition to avoid potentially fatal haemoptysis and in providing detailed anatomical localisation to guide further management. Endovascular embolisation using coils or plugs is considered the treatment of choice and preferred over open surgical repair as it is less invasive and has fewer complications. ${ }^{1,2}$ In the case of our patient, it achieved desired pseudoaneurysm occlusion with subsequent resolution of her symptoms.

\section{References}

1 Chen Y, Gilman MD, Humphrey KL et al. Pulmonary artery pseudoaneurysms: clinical features and CT findings. Am J Roentgenol 2017;208:84-91.

2 Valente T, Abu-Omar A, Sica G et al. Acquired peripheral pulmonary artery aneurysms: morphological spectrum of disease and multidetector computed tomography angiography findings-cases series and literature review. Radiol Med 2018;123:664-75.

3 Agarwal PP, Dennie CJ, Matzinger FR, Peterson RA, Seely JM. Pulmonary artery pseudoaneurysm secondary to metastatic angiosarcoma. Thorax 2006;61:366.

Address for correspondence: Dr Divya Vaid, Department of Radiology, Addenbrooke's Hospital, Cambridge University Hospitals NHS Foundation Trust, Hills Road, Cambridge CB2 OQQ, UK.

Email: divya.vaid@addenbrookes.nhs.uk 